\title{
Graded assessment and classification of impaired temperature sensibility in patients with diabetic polyneuropathy
}

\author{
Per Hansson, Ulf Lindblom, Per Lindström
}

\begin{abstract}
Thermal sensibility was quantitatively assessed in the feet of 46 diabetic patients. In subjects with sensibility deficits the perception threshold for warmth or cold, or of heat pain, was either increased or lost. Four stages of impaired thermal sensibility were defined, and a classification of dysfunction is proposed which could be useful in routine clinical examination of patients with diabetic polyneuropathy. The classification of impaired thermal sensibility correlated significantly with the results of a bedside screening examination aimed at describing the severity of the polyneuropathy in terms of its regional extent.
\end{abstract}

Diabetic polyneuropathy sometimes involves only large or small nerve fibres selectively, but in the majority of patients, disturbances in function and morphology of fibres of all diameters are present. ${ }^{1}$ Clinical assessment of function in different nerve fibres is routinely performed with standardised bedside examination techniques and, for sensibility, sometimes with quantitative methods. ${ }^{2}$ Sensory large fibre function may be examined using neurophysiological techniques for conduction velocity measurement. It is not possible to monitor the function of small myelinated and unmyelinated fibres using such methods. A recent technique for examining functions subserved by these fibres is the Marstock method $^{3}$ for quantitative thermal stimulation.

In this study quantitative thermal testing was performed in the feet of patients with diabetes, and a classification of impaired thermal sensibility is proposed which could be useful in cross-sectional and long term studies of patients with diabetic polyneuropathy. The classification included threshold variations and aberrant phenomena such as paradoxical sensation and fatigue which may confuse the less experienced observer. ${ }^{4}$ It was not the aim of this study to present data on the relative frequency of different abnormalities.

Neurology, Karolinsk

Hospital, Stockholm

$P$ Hansson

U Lindblom

$P$ Lindström

Correspondence to: $\mathrm{Dr}$ Hansson, Department of Neurology, Karolinska Hospital, 10401 Stockholm, Sweden

Received 7 March 1990 and in final revised form 12 November 1990

Accepted 22 November 1990

\section{Methods}

Thermal sensibility has been assessed quantitatively in the feet of 46 consecutive diabetic patients referred to our department for evaluation of suspected disturbances in peripheral nerve function. There were 14 females (42-68 years of age, mean 53.8) and 32 males (23-71 years of age, mean 53.6). Duration of diabetes was two to 51 years with a mean of 15.5 years. Five patients were on oral antidiabetic medication and 41 were on insulin. None of the "patients suffered from renal dysfunction or were addicted to alcohol.

The neurological investigation included a clinical bedside examination with a fixed protocol (table 1). Sensory screening was carried out using a camel hair brush for touch, and figure writing with a blunt pencil for tactile discrimination on finger pads, the dorsum of the feet and lower legs. Normally, figures of less than $1 \mathrm{~cm}$ height are recognised on the finger pads and figures 4-6 cm high should be correctly identified on the legs and feet. Pinprick with a disposable pin was used for mechanically evoked pain, and two metallic rollers kept at 20 and $40^{\circ} \mathrm{C}$, respectively, for cold and warmth. The results of hypo- or hyperaesthesia for touch, figure writing, pinprick, temperature or weakness and atrophy were graded according to their spatial distribution as were reflex dysfunction (table 1). A single-sided score of 1 was recorded as 0 , while higher asymmetrical scores

\begin{tabular}{|c|c|c|c|c|}
\hline \multicolumn{3}{|c|}{ SIGNS RIGHT SIDE } & LEFT SIDE & Grade \\
\hline \multicolumn{2}{|c|}{ TOUCH } & 1 & 1 & 1 \\
\hline \multicolumn{2}{|c|}{ TOUCH DISCRIMINATIVE } & 2 & 1 & 1.5 \\
\hline \multicolumn{2}{|c|}{ PINPRICK } & 2 & 2 & 2 \\
\hline \multicolumn{2}{|c|}{ COLD } & 2 & 2 & 2 \\
\hline \multicolumn{2}{|c|}{ WARMTH } & 2 & 2 & 2 \\
\hline \multicolumn{2}{|c|}{ PARESIS } & 0 & 0 & 0 \\
\hline \multicolumn{2}{|c|}{ ATROPHY } & 0 & 0 & 0 \\
\hline \multirow{3}{*}{$\underset{\underset{\alpha}{\vec{u}}}{\vec{u}}$} & BICEPS & + & + & \multirow[b]{3}{*}{1} \\
\hline & QUADRICEPS & + & + & \\
\hline & GASTROC-SOL & - & - & \\
\hline \multicolumn{3}{|c|}{ 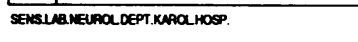 } & \multicolumn{2}{|c|}{ SUM OF GRADEs $9 \cdot 5$} \\
\hline
\end{tabular}

Signs of polyneuropathy with numerical estimation of severity

Types of function or sign

Touch defection

0 If unilateral

1 If restricted to

Pinprick

Warmth

Paresis

Muscle atrophy

the feet

2 If extending to the

lower legs

3 If also in the upper extremitios
Muscle reflexes

(ankle, kneee and biceps brachii)

0 For unilateral loss

1 For loss of one on

each side

2 For loss of 3.4

reflexes

For loss of 5-6

Table Protocol and instructions for recording signs of sensory-motor disturbances during bedside screening examination. 
Figure 1 Recording of normal thermal thresholds from the feet of a patient. The cold pain threshold (CPT) could not be determined since pain was not evoked even at a

temperature of about $7^{\circ} \mathrm{C}$. Reference limits set as a basis for the presented classification of impaired thermal sensibility is seen to the left $40^{\circ} \mathrm{C}$ and $45^{\circ} \mathrm{C}$ were the upper limits set for warm threshold (WT) increase and loss of warm sense, respectively. A heat pain threshold (HPT) above $50^{\circ} \mathrm{C}$ was considered as loss of this sense. $27^{\circ} \mathrm{C}$ was the limit set for cold threshold (CT) increase, and $10^{\circ} \mathrm{C}$ for loss of cold sense.

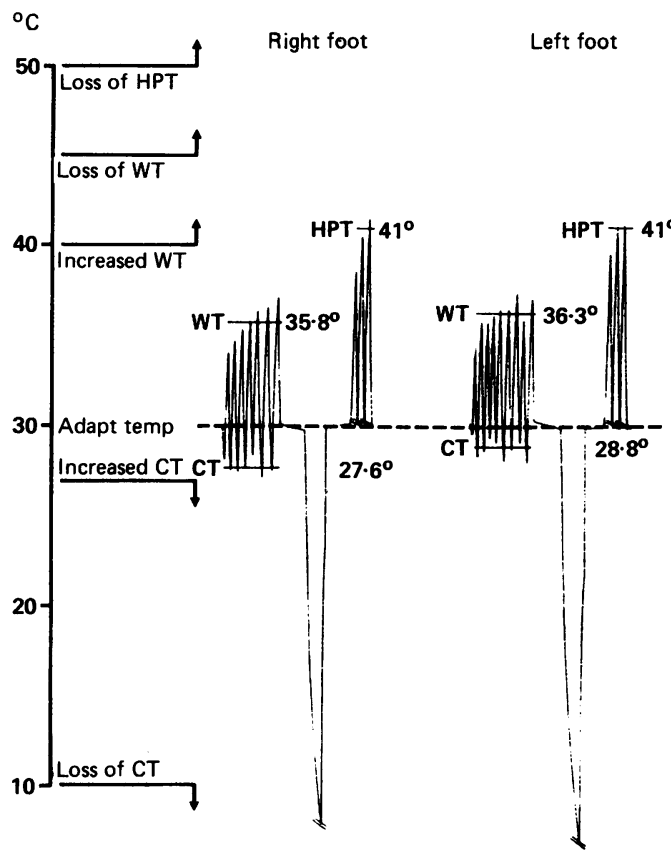

were averaged. The average score for each function was then added to provide a total neuropathy score (maximum 24).

This study did not aim to characterise neuropathic symptoms and these are therefore not reported.

Quantitative thermal testing was performed,

${ }^{\circ} \mathrm{C}$

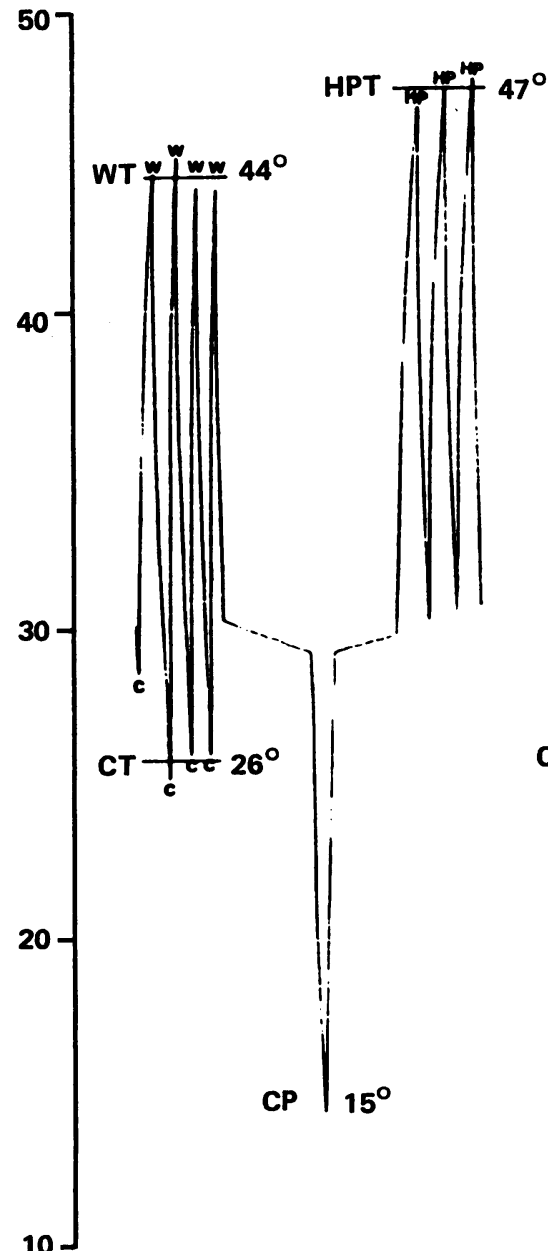

Figure 2 For details see text. $W=$ perception of warmth, $C=$ perception of cold, $H P$ $=$ perception of heat pain. $C P=$ perception of cold pain. For other abbreviations see fig

\section{Left foot}
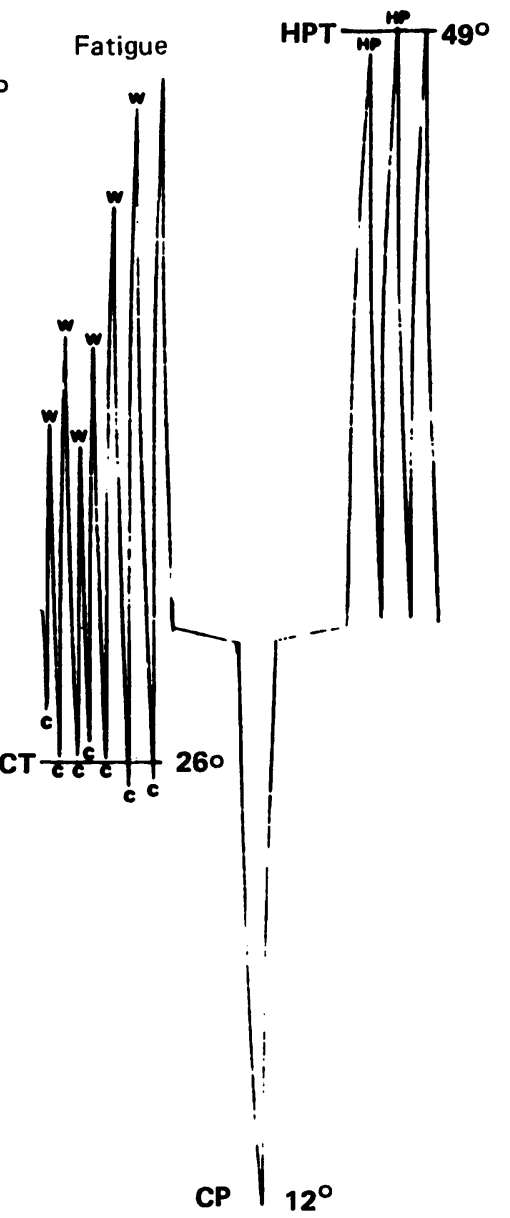
1 

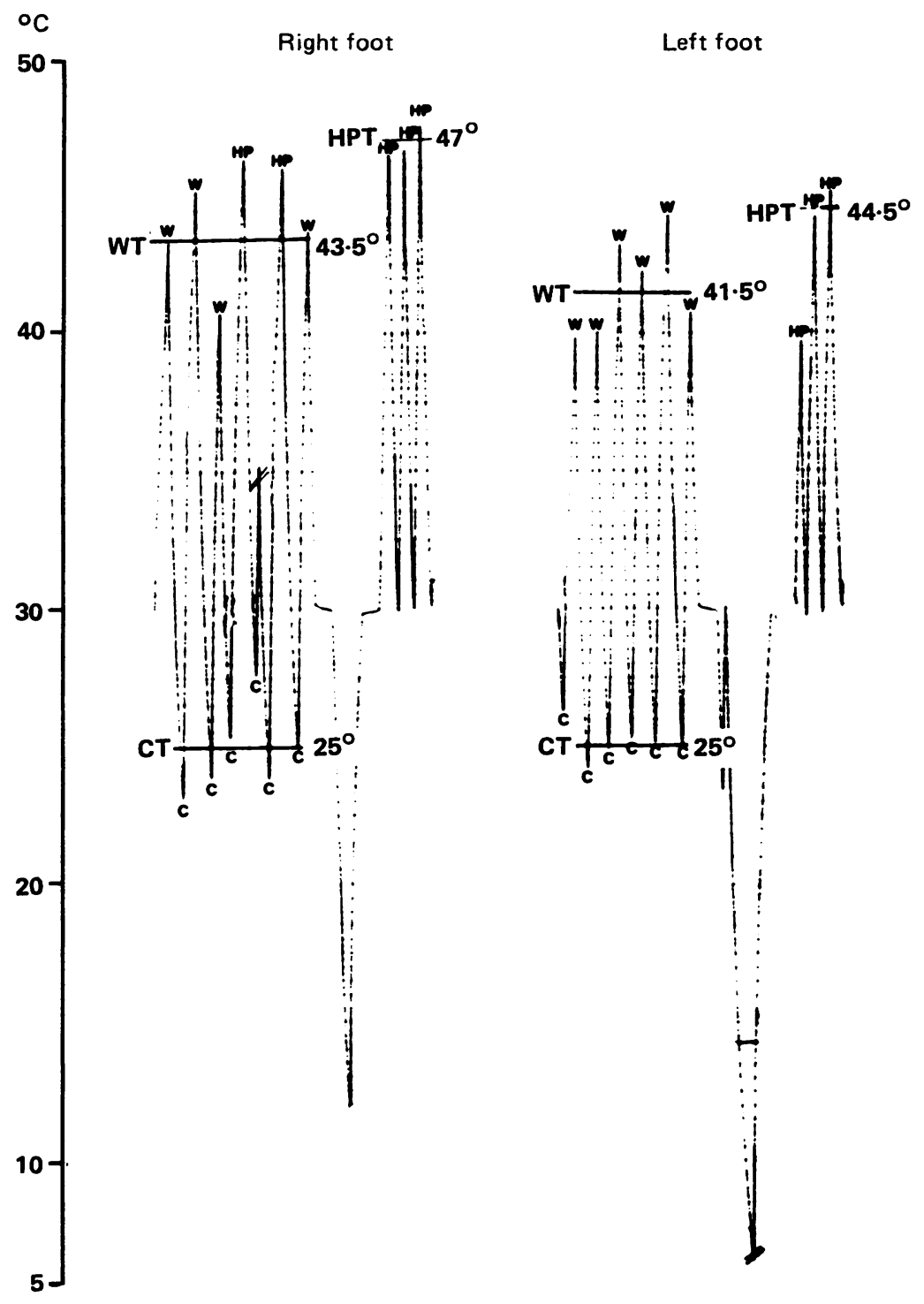

Figure 3 For details see text. Abbreviations as fig 1 and 2.

right foot. This finding shows the need for repeated verbal communication with the patient during the recording session concerning the character of the experienced sensation. A record with perception level variations of more than $\pm 2^{\circ} \mathrm{C}$ was defined as irregular threshold. The cold threshold was increased to about $25^{\circ} \mathrm{C}$ in both feet and was in addition irregular in the right foot.

Paradoxical sensation, that is, a sensation of cold during application of a warm stimulus, or vice versa, was found in some patients which, again, underlines the importance of repeated verbal communication with the patient.

Fatigue, appearing as successively increasing perception levels, is demonstrated in fig 2 by the recording of warm perception in the left foot. In this particular patient it was crucial to continue the recordings after the first four measurements of warm perception to be able to detect the fatigue phenomenon.

Loss of either warm or cold sense, or both, is exemplified in fig $4 \mathrm{~A}$ by a recording from the left foot of a patient with only preserved heat pain perception. At cold stimulation of about 5$10^{\circ} \mathrm{C}$ no sensation or paradoxical warmth was reported.
Thermal anaesthesia, that is, no temperature sensation in the stimulus range of $5-50^{\circ} \mathrm{C}$, is illustrated in the recording from the left foot of a patient in fig $4 \mathrm{~B}$, who reported only faint warmth at an unphysiological level of about $55^{\circ} \mathrm{C}$.

Classification according to severity

Based on the results of this study we propose the following classification of impaired thermal sensibility, in ascending severity:

Class $O$ : Normal sensibility.

Class 1: Increased threshold to warmth, that is, above $40^{\circ} \mathrm{C}$ and below $45^{\circ} \mathrm{C}$ and/or increased threshold to cold, that is, below $27^{\circ} \mathrm{C}$ but above $10^{\circ} \mathrm{C}$. Irregular threshold, which was found in only two patients, is also included in this class. Class 2: Loss of warm sense, that is, the threshold exceeded $45^{\circ} \mathrm{C}$, or loss of cold sense defined as a threshold below $10^{\circ} \mathrm{C}$. Paradoxical sensation (three patients) and fatigue (four patients) were classified as loss of threshold.

Class 3: Loss of warm and cold thresholds and only a heat pain threshold below $50^{\circ} \mathrm{C}$ left.

Class 4: Thermal anaesthesia, that is, loss of warm and cold threshold combined with a heat pain threshold exceeding $50^{\circ} \mathrm{C}$.

If the feet of a particular patient were differentially classified (see fig 2) an average was calculated.

Significant correlations were found when comparing the classification outcome with the results of the clinical bedside examination of small fibre function $\left(r_{s}=0.39, p<0.01\right)$, sen-

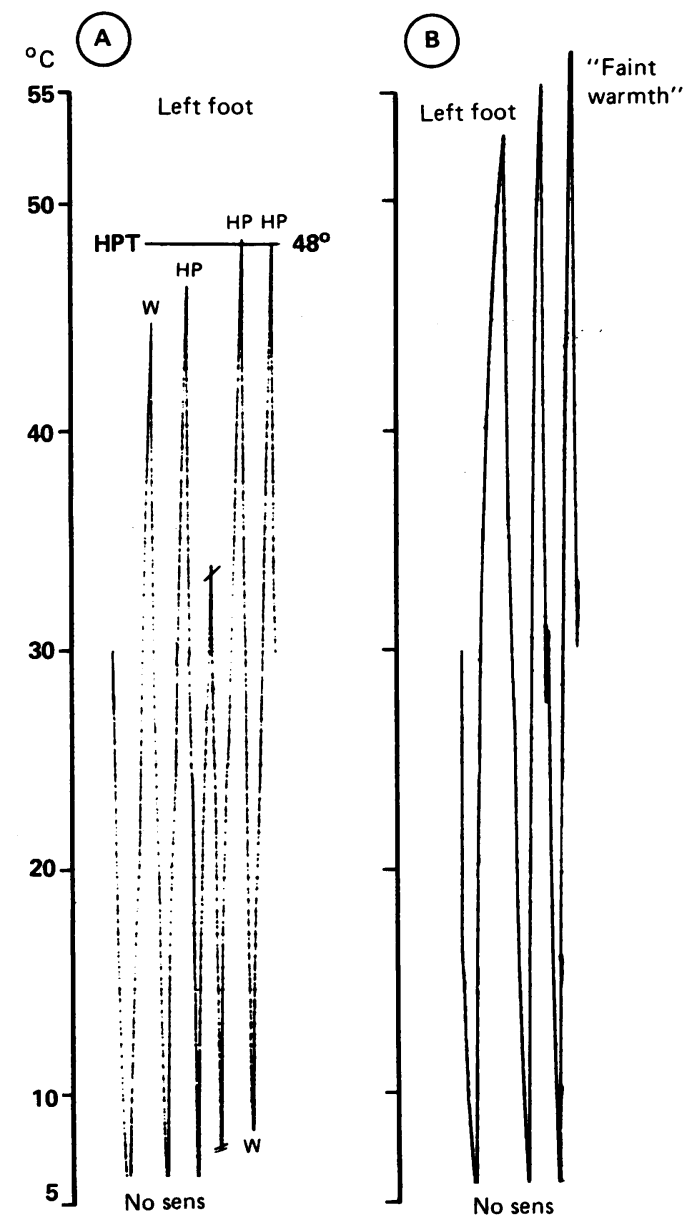

Figure $4 A$ and $B$. For details see text. Abbreviations as fig 1 and 2 . 
sory large fibre function $\left(r_{s}=0.85, p<0.001\right)$, and total neuropathy score $\left(r_{s}=0.54\right.$, $p<0.001)$. In a few patients, however, we found sensibility dysfunction on clinical examination of small fibre function (suprathreshold stimuli) but normal thermal thresholds, and vice versa.

Nine feet of seven patients were categorised in class $0-2$ in spite of having a HPT above $50^{\circ} \mathrm{C}$ (only one foot in class $\mathrm{O}$ ).

Total duration of thermal threshold measurements did not exceed 20 minutes in any of the patients.

\section{Discussion}

Physiologically it is possible to relate threshold increase and loss of thermal senses in patients with diabetes to different proportions of axonal degeneration in subsets of nerve fibres, that is, C-fibres for warmth and heat pain, and A-deltafibres for cold and possibly heat pain. In A-delta fibres demyelination may also contribute to the dysfunction. Whether our findings of irregular threshold, paradoxical sensation and fatigue reflect different degrees of peripheral and/or central functional and morphological aberration is not known. Paradoxical sensation and fatigue may represent an intermediate, less severe, impairment than loss of threshold but were classified as loss in the light of the definition of threshold (see Methods). Irregular threshold, on the other hand, was classified as threshold increase and possibly represents a less severe abnormality than paradoxical sensation and fatigue. Since only nine patients reported either of these three phenomena the material was too small to enable a rank correlation with the severity of polyneuropathy based on the clinical findings. All nine patients scored a minimum of seven points in the total polyneuropathy score.

When evaluating the significant correlations found between the proposed classification of impaired thermal sensibility and the clinical scores, it must be emphasised that we compared the outcome of a quantitative thermal test in the feet with the results of a clinical bedside examination aimed at describing the regional extent of disturbed sensibility. These findings, however, form the basis for two essential conclusions. First, they support the proposed order of precedence of the classification. Secondly, the correlation with sensory large fibre function argues in favour of the conclusion that selective affection of large or small fibre function was not the rule in the majority of patients. Subsets of patients may, however, demonstrate either small or large fibre dysfunction. This interpretation derives support from the results of Ziegler et al, ${ }^{7}$ who found that sensory nerve conduction velocity in the arms and legs as well as malleolar vibration sensibility correlated significantly with thermal non-nociceptive sensibility in the feet. Our findings of a few patients with normal thermal thresholds but dysfunction regarding the sensation of suprathreshold stimuli activating small fibres, and vice versa, emphasises the importance of assessing both threshold and suprathreshold sensibility to assure that deficiencies in small fibre function do not escape detection.

Seven patients reported a HPT above $50^{\circ} \mathrm{C}$ in one or both feet but, regardless of this, were all categorised in class $0-2$ based on other criteria. Categorisation of the HPT could perhaps have increased the sensitivity of the classification but, due to the large interindividual variations in HPT, this probably also would have induced a decrease in specificity and was not included. In addition, Ziegler $e t a l^{8}$ have demonstrated a lack of differences in HPT in the feet of diabetics and matched controls.

In the presented classification the cold pain threshold has on purpose been disregarded due to extremely large interindividual variations. In addition, a fraction of normal subjects do not experience cold pain even at temperatures as low as $5^{\circ} \mathrm{C}$. It is still important to test for $\mathrm{CP}$ to detect qualitative abnormalities like dysaesthesia and allodynia as well as temporo-spatial abnormalities such as after-sensation, and faulty localisation. ${ }^{9}$ Since these abnormalities, whether evoked by warm or cold stimulus application, could be centrally mediated they may give clues to whether peripheral and/or central factors should be the target for treatment of neuropathic pain, which sometimes accompany these patients. Except for the three patients reporting paradoxical sensation no qualitative abnormalities were found. The lack of allodynic responses agrees with the results from Ziegler et al.

The proposed classification of impaired thermal sensibility, which reflects the severity of the polyneuropathy in terms of its regional extension could be useful in cross-sectional and long-term studies of patients with diabetic polyneuropathy.

We thank Mrs Berit Lindblom for her technical assistance and patient care. The study was supported by grants from the Karolinska institute and the Swedish Association for the Neurologically Disabled (NHR).

1 Thomas PK, Eliasson SG. Diabetic neuropathy. In: Dyck PJ, Thomas PK, Lambert EH, Bunge R, eds. Peripheral neuropathy, vol 2. Philadelphia: W B Saunders, Peripheral neuropat

2 Lindblom $U$. Clinical and instrumental diagnostic approaches to sensory disturbances in diabetic peripheral neuropathy. In: J P Assal, et al, eds. Diabetes research and clinical practice, vol 2. Amsterdam: Elsevier, 1986:213-25.

3 Fruhstorfer H, Lindblom U, Schmidt WG. Method for quantitative estimation of thermal thresholds in patients. J Neurol Neurosurg Psychiatry 1976;39:1071-5.

4 Liniger C, Assal J-P. Quantitation of sensory deficits. Reproducibility and interpretation in diabetic patients with neuropathy; some unanswered questions. In: J-P Assal, C Linger, eds. Peripheral neuropathies 1988. What is significantly new? Fida research series Vol 21. Padua; Liviana Press, Springer Verlag, 1989:145-56.

5 Konietzny F. Peripheral neural correlates of temperature sensations in man. Human Neurobiology 1984;3:21-32.

6 Habermann-Horstmeier L. Altersbedingte schwellenänderungen der cutanen vibrations-, temperatur- und schmerzempfingen der cutanen vibrations-, temperatur-und schmerzempfin-
dung des menschen. Thesis. (Institut fur normale und dung des menschen. Thesis. (Institut fur normale und
pathologische physiologie der Philipps-Universität Marpathologische phy

7 Ziegler D, Mayer P, Wiefels K, Gries A. Assessment of small and large fibre function in long term type 1 (insulindependent) diabetic patients with and without painful neuropathy. Pain 1988;34:1-10.

8 Ziegler D, Mayer P, Gries FA. Evaluation of thermal, pain, and vibration sensation thresholds in newly diagnosed Type 1 diabetic patients. J Neurol Neurosurg Psychiatry 1988;51:1420-4.

9 Lindblom U. Assessment of abnormal evoked pain in neurological pain patients and its relation to spontaneous pain: A descriptive and conceptual model with some analytic results. In: Fields HL, et al, eds. Advances in pain research and therapy, Vol 9. 1985:409-23. 